\title{
Intraretinal Exudates in Coats' Disease as Demonstrated by Spectral-Domain OCT
}

\author{
Rivka Kessner Adiel Barak Meira Neudorfer \\ Department of Ophthalmology, Tel Aviv Medical Center, Tel Aviv, Israel
}

\section{Key Words}

Coats' disease - Intraretinal exudates · Outer plexiform layer - Spectral-domain optical coherence tomography

\section{Abstract \\ Purpose: To describe the spectral-domain optical coherence tomography (SD-OCT) findings in a case of Coats' disease, with emphasis on the intraretinal exudates.}

Methods: Case report of a 4.5-year-old girl who presented with total exudative retinal detachment and organized exudates in a stellate configuration. SD-OCT was performed before and after treatment, which included surgical drainage of subretinal fluid and indirect laser application during the surgery.

Results: At presentation, the SD-OCT showed an elevation of the foveal contour, with thickening of the retina, many exudates and a large quantity of subretinal fluid. Two months after the surgical treatment, SD-OCT revealed a significant reduction in the amount of subretinal fluids, but with persistence of the exudates.

Conclusion: SD-OCT can be used as an important tool in order to describe the changes in each layer of the retina in Coats' disease. Considering the histopathological findings in Coats' disease, it is reasonable to assume that the exudates accumulate in the outer plexiform layer.

\section{Introduction}

Coats' disease is a rare idiopathic condition, characterized by telangiectatic retinal vessels, with chronic accumulation of intraretinal and subretinal exudates. In most cases, the disease progresses slowly, with increasing exudative retinal detachment. It is usually unilateral and occurs mostly in children [1].

Histopathological examinations of eyes with Coats' disease show thickening of the sensory retina by homogeneous acellular material, typical of lipoproteinaceous exudation. Lipid- and hemosiderin-laden macrophages and cholesterol clefts are 
present in both the sensory retina and subretinal fluid. Lipid deposition induces foreign-body granulomatous inflammation [2].

Unlike other diseases which involve intraretinal exudates, the precise location of the exudates in Coats' disease has not been emphasized in the literature. In the last decade, optical coherence tomography (OCT) has been commonly used to describe the characteristic findings in Coats' disease [3]. Yet, there seems to be a lack of studies on spectral-domain OCT (SD-OCT) in Coats' disease.

The purpose of the present study is to describe SD-OCT findings in Coats' disease, with emphasis on the intraretinal exudates.

\section{Case Report}

A 4.5-year-old girl, who had a 2-week history of lateral deviation of her right eye, was referred to the Tel Aviv Medical Center clinic. On fundus examination of her right eye, she had total exudative retinal detachment with tortuous vessels, micro-aneurysms, and organized exudates in stellate configuration. The fundus examination of her left eye was normal. Fluorescein angiography showed telangiectatic vessels and aneurysms in the superior-temporal area (fig. 1). SD-OCT was performed using the Spectralis (Heidelberg Engineering, Vista, Calif., USA). It showed flattening of the foveal contour, cystic hydration of the neurosensory retina, and relative thinning of the perifoveal area. The tissue appeared disorganized and there was loss of the normal retinal layering; additionally, many hyperreflective foci (the exudates) were seen in the neuroretina. It was difficult to conclusively identify in which layer the exudates were found, but it appeared that they were concentrated mainly in the outer layers and the border of the serous detachment (fig. 2 ). Two intravitreal injections of 0.05 $\mathrm{ml}$ Avastin (1.25 mg bevacizumab) to the patient's right eye and peripheral cryotherapy were performed - without any significant improvement in her visual acuity or fundus examination. A subretinal fluid drainage was performed, with indirect laser applications to the non-perfused areas during the surgery. There was improvement of vision from light perception to $1 / 60$ and significant improvement of subretinal fluids, with minimal persistent elevation of the foveal area. Two weeks after the surgery, marked absorption of the subretinal fluid was seen. An SD-OCT, done 2 months after the surgery, showed a significant decrease in the retinal thickness, but with the persistence of intraretinal exudates (fig. 3). The patient continues to be followed up by our retina service.

\section{Discussion}

Histopathological studies on Coats' disease describe heavy infiltration of the retina with foam cells and 'ghost cells', particularly in the inner layers - and intensely eosinophilic exudates in some parts of the outer retina. These pathological changes completely disrupt the normal architecture of the retina [4].

Coats' disease can be compared to diabetic retinopathy, since hard retinal exudates are often seen in eyes with diabetic macular edema (DME). In DME, the hard exudates have been reported to be composed of lipid and proteinaceous material, and the exudates are deposited primarily in the outer plexiform layer of the retina [5]. The accumulation of hard exudates in the outer retina might be explained by the theory of the mechanisms of fluid movement in the retina as proposed by Marmor [6]. He reported that the external limiting membrane (ELM), which consists of the zonula adherens between Müller cells and photoreceptors at the base of the outer segments, has a very narrow channel. Unlike the zonula occludens of the retinal pigment epithelium and retinal capillaries, the ELM channel particularly limits the movement of 
large molecules. Accordingly, a breakdown of the blood-retina barrier in eyes with diabetic retinopathy could cause extravasation of lipids and proteins. However, because these exudates could not pass through the ELM owing to their large size, they accumulated anterior to the ELM, resulting in swelling in the outer retina [7]. This theory has not been attributed to Coats' disease in the literature, though the breakdown of the blood-retina barrier mechanism is similar.

Another disease that involves macular edema with accumulation of exudates is catscratch disease neuroretinitis. A recent publication described the OCT findings of eyes with macular edema secondary to cat-scratch disease neuroretinitis and showed the same localization of the exudates as in our case (outer plexiform layer) [8].

SD-OCT provides improved image quality as well as 3-dimensional imaging of the retina by shortening the time over which images are acquired by 25- to 100 -fold, compared with time-domain OCT (TD-OCT). With SD-OCT, the enhanced definition of the retinal layers and fine photoreceptor structures allows for precise localization of intraretinal lesions in each retinal layer. Hard exudates usually appear as hyperreflective dots [9]. TD-OCT, on the other hand, supplies only little information about the different layers of the retina.

Several case reports regarding Coats' disease have shown TD-OCT findings of the disease. All of these studies focused on the calculation of the central retinal thickness, and on the demonstration of macular edema and serous retinal detachment. Few of these studies have shown the macular subretinal and intraretinal exudates, yet have not related to the intraretinal exudates $[3,10]$. To our knowledge, there are no studies in the literature discussing the location of the intraretinal exudates in Coats' disease, using TD-OCT.

In the above case report of a patient with Coats' disease, we demonstrated intraretinal exudates - most probably mainly in the outer plexiform layer of the retina, using SD-OCT. This appearance in OCT has not been previously discussed regarding Coats' disease. We also showed the changes in SD-OCT during the clinical course of the patient's illness and the significant reduction in the retinal thickness after surgical treatment, but with persistence of the exudates.

In a study about the effect of photocoagulation on intraretinal lipid exudates in DME, using SD-OCT, multiple hyperreflective foci were found mainly in the outer nuclear layer, but also in the outer plexiform and inner nuclear layers. Months after laser treatment, the hard exudates had resolved partially in the OCT and the corresponding hyperreflective conglomerates had become smaller [11].

Considering the histopathological findings in Coats' disease, it is reasonable to assume that the exudates accumulate in the outer plexiform layer. We believe that SDOCT can be used as an important tool in order to describe the changes in each layer of the retina in Coats' disease, as in other diseases. Obviously, more SD-OCT results from patients with Coats' disease are needed in order to strengthen our assumptions.

\section{Disclosure Statement}

The authors received no financial support for this paper and have no conflicts of interest to declare. 


\section{Case Reports in Ophthalmology}

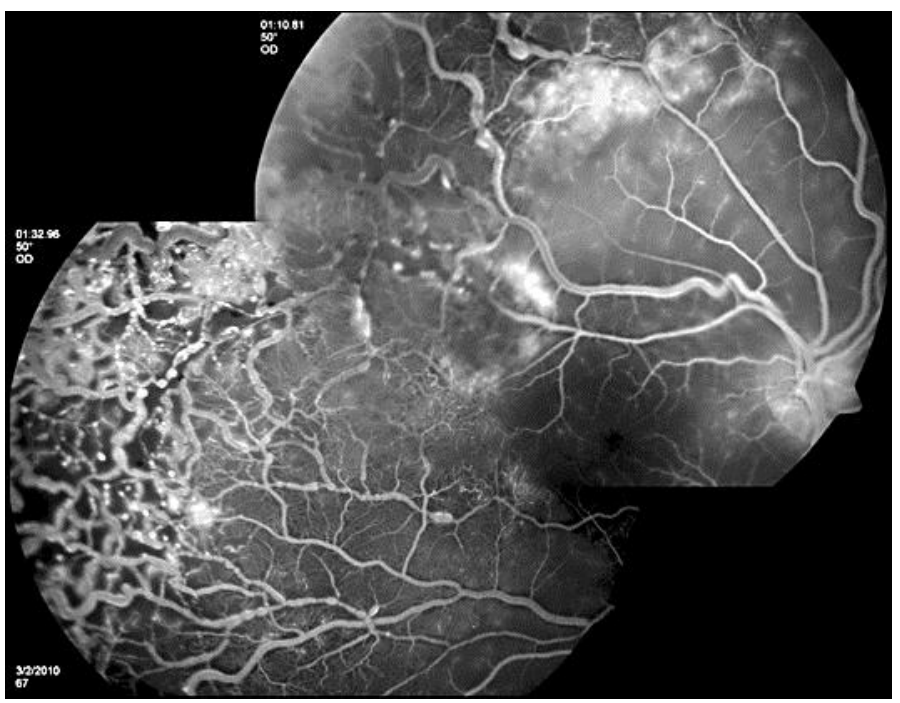

Fig. 1. Fluorescein angiography of the right eye at presentation reveals telangiectatic vessels with leakage in the superior-temporal area and macro-aneurysms.
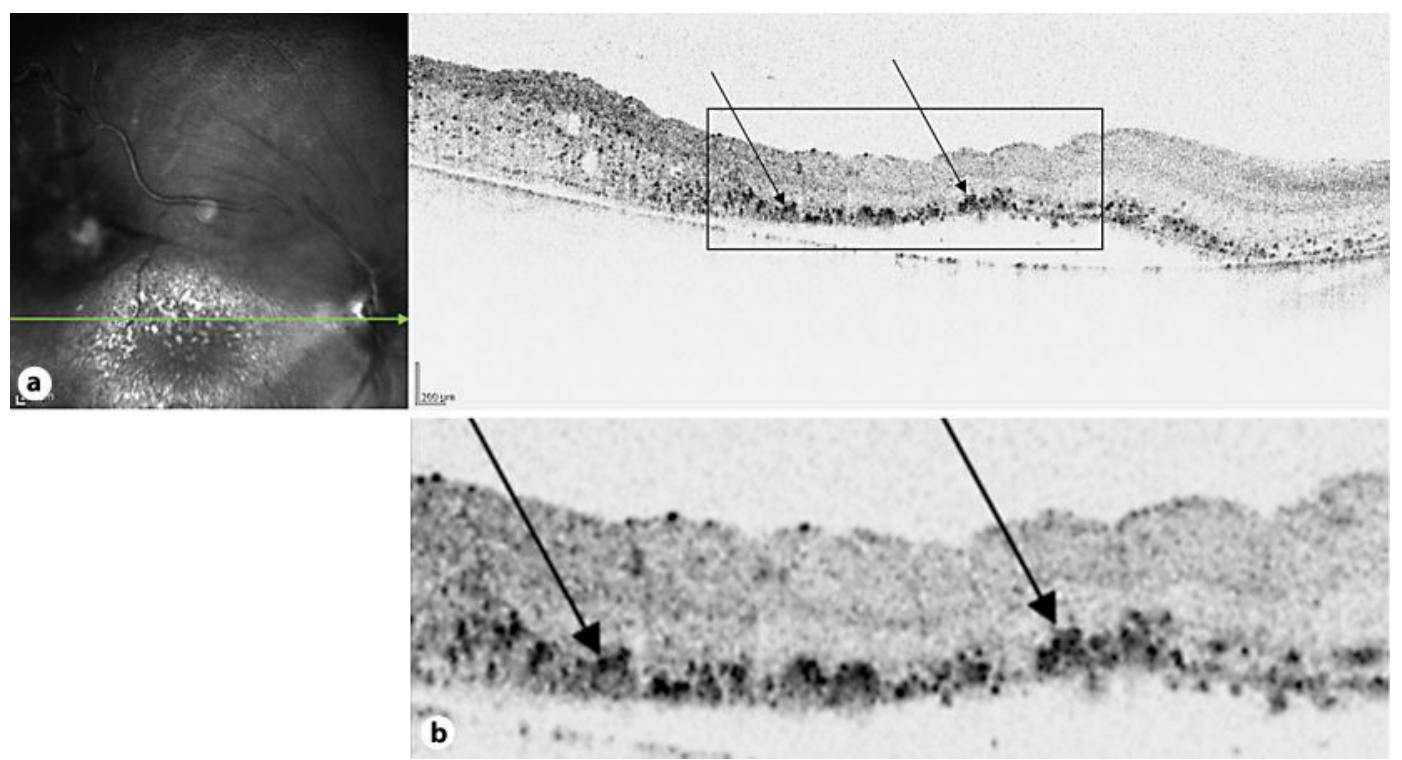

Fig. 2. SD-OCT imaging of the right eye at presentation, using the Spectralis (Heidelberg Engineering). A cross scan through the macula shows diffuse thickening of the retina, a large quantity of subretinal fluid, and many intraretinal exudates (arrows) - mainly in the outer plexiform layer. 


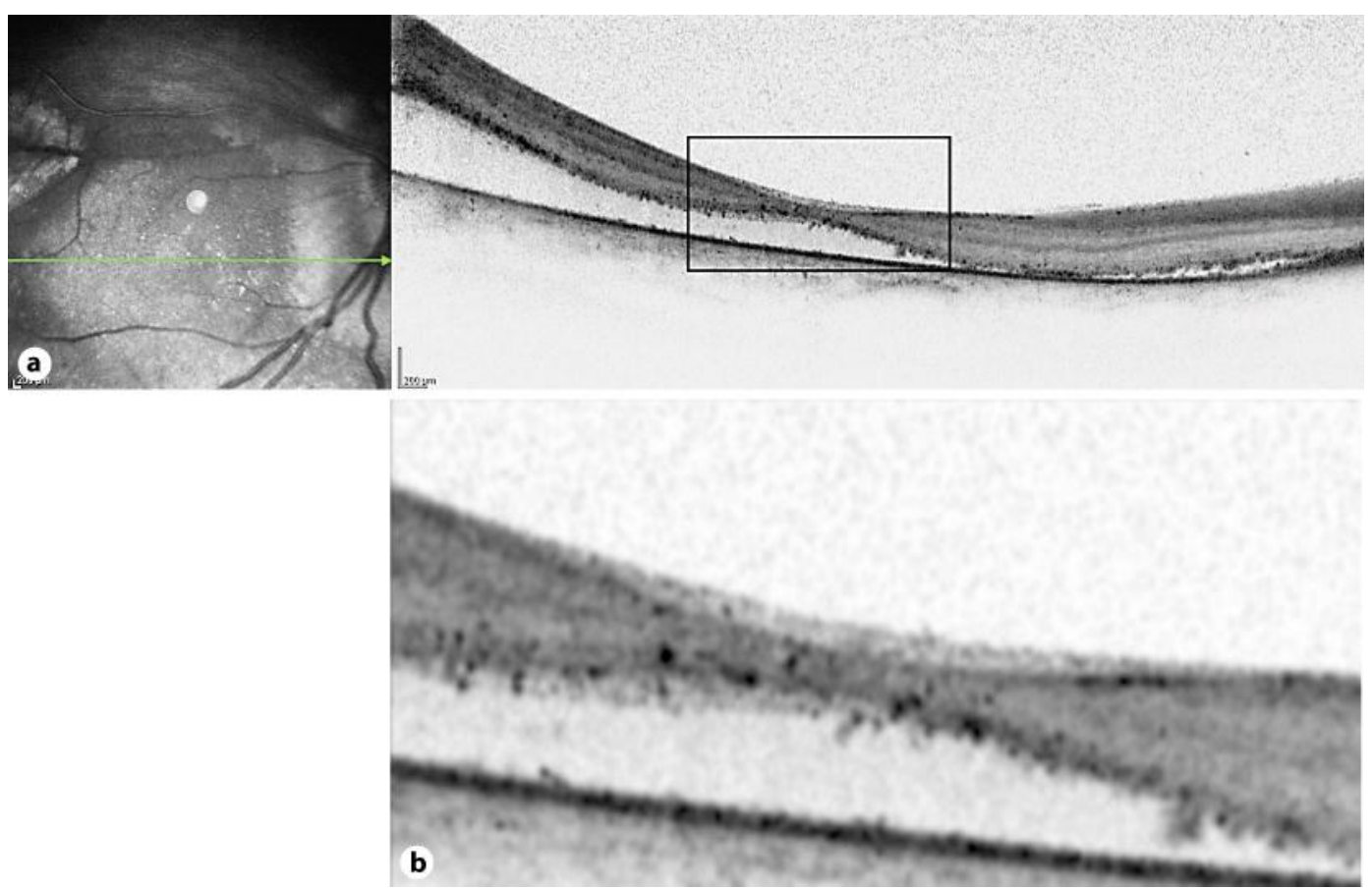

Fig. 3. SD-OCT imaging of the right eye performed 2 months after surgical treatment shows a significant reduction in the retinal thickness and a mild decrease in the amount of exudates.

\section{References}

1 Shields JA, Shields CL, Honavar SG, Demirci H, Cater J: Classification and management of Coats' disease: the 2000 Proctor Lecture. Am J Ophthalmol 2001;131:572-583.

2 Chang MM, McLean IW, Merritt JC: Coats' disease: a study of 62 histologically confirmed cases. J Pediatr Ophthalmol Strabismus 1984;21:163-168.

-3 Lin CJ, Hwang JF, Chen YT, Chen SN: The effect of intravitreal bevacizumab in the treatment of Coats' disease in children. Retina 2010;30:617-622.

-4 Tripathi R, Ashton N: Electron microscopical study of Coats' disease. Br J Ophthalmol 1971;55:289-301.

5 Otani T, Kishi S: Tomographic findings of foveal hard exudates in diabetic macular edema. Am J Ophthalmol 2001;131:50-54.

-6 Marmor MF: Mechanisms of fluid accumulation in retinal edema. Doc Ophthalmol 1999;97:239-249.

-7 Ota M, Nishijima K, Sakamoto A, Murakami T, Takayama K, Horii T, Yoshimura N: Optical coherence tomographic evaluation of foveal hard exudates in patients with diabetic maculopathy accompanying macular detachment. Ophthalmology 2010;117:1996-2002.

-8 Habot-Wilner Z, Zur D, Goldstein M, Goldenberg D, Shulman S, Kesler A, Giladi M, Neudorfer M: Macular findings on optical coherence tomography in cat-scratch disease neuroretinitis. Eye (Lond) 2011;25:1064-1068.

-9 Sakamoto A, Hangai M, Yoshimura N: Spectral-domain optical coherence tomography with multiple Bscan averaging for enhanced imaging of retinal diseases. Ophthalmology 2008;115:1071-1078.

10 Stergiou PK, Symeonidis C, Dimitrakos SA: Coats' disease: treatment with intravitreal bevacizumab and laser photocoagulation. Acta Ophthalmol 2009;87:687-688.

11 Deák GG, Bolz M, Kriechbaum K, Prager S, Mylonas G, Scholda C, Schmidt-Erfurth U; Diabetic Retinopathy Research Group Vienna: Effect of retinal photocoagulation on intraretinal lipid exudates in diabetic macular edema documented by optical coherence tomography. Ophthalmology 2010;117:773-779. 